\title{
PSYCHOLOGICAL WILLINGNESS FOR MATERNITY AS A FACTOR CONTRIBUTING TO THE HEALTHY BEHAVIOR OF WOMEN
}

\author{
Ekaterina A. Burina ${ }^{1}$, \& Valentina A. Moshkivskaya ${ }^{2}$ \\ ${ }^{1}$ St. Petersburg State University (Russia) \\ ${ }^{2}$ St. Petersburg Maternity Hospital №18 (Russia)
}

\begin{abstract}
The health protection of a child and a mother is one of the priority tasks with reproductive health of a woman being central. Thus, identifying the factors that have a negative impact on reproductive activity is of a great importance. The aim of the research is to study the psychological willingness for motherhood (conscious motherhood) in connection with the health-saving behavior of pregnant women. This study is one of the first in the Russian sample, examining various aspects of health-saving behavior during pregnancy. The selected and developed materials and methods of research take into account the peculiarities of the state of the pregnant woman. Realization of applied value in practice is the implementation of preventive measures (active and passive informing of women) at each stage and further evaluation of the effectiveness of the measures implemented. A total of 100 pregnant women of childbearing age take part in the study. Pregnant women do not have full correct knowledge and adequate attitudes about aspects of health-saving behavior, thereby demonstrating problem behavior (alcohol, smoking, the risk of alcohol-exposed pregnancy, that is, the risk of fetal alcohol syndrome). Under the influence of the implemented preventive measures, the behavior of pregnant women becomes more health-saving, knowledge and attitudes in this area change. Preventive materials developed in the framework of the project and implemented measures prove their effectiveness and can be recommended for application in the field of health related to the reproductive sphere of women. The study is supported by the RFBR grant № 20-013-00759a.
\end{abstract}

Keywords: Conscious motherhood, willingness for maternity, pregnancy, health-saving behavior, prevention.

\section{Introduction}

Nowadays in world science there is a tendency to increase the number of studies devoted to the research of health saving behavior in the reproductive sphere (Innamorati M., Sarracino D., Dazzi N., 2010; Slade et al., 2005; Chiu-Mieh Huang et al., 2011; Suena H.M., et al., 2017; Atkinson L., Shaw R.L. et al., 2011; Williams et al., 2011; Lawrence W.T., Haslam C., 2007; Judith H.M., John B.L.; Stene-Larsen K. et al., 2009; Gajewska E., Mojs E., 2008; Andersen M.R. et al., 2009; Balachova T.N., 2008; Borisenko L.V., 2003; Yeremkina E.P., Tyurina N.A., 2015; Shcherbakov A.V., Vasilyeva E.N., 2015; Zhuravlev A.L., 2004; Olfert V.V., 2004). At the same time, in part of the research, trends in risk behavior, leading to potential impairments and complicated pregnancy, are considered first (Gray J. et al., 2005; Morrison A. et al., 2007; Teasdale C.A., Abrams E.J. et al., 2017).

Moreover, the study of maternal awareness, psychological readiness for motherhood, and its influence on behavior in the reproductive and health-saving sphere is actively developing. Pregnancy can be a moment for positive change in health behavior, since this is a period when women are motivated for health and are in constant contact with health care professionals. It is assumed that raising awareness of the mother during pregnancy can be an effective way to improve her health as well as the health of the newborn (Akerjordet K., Severinsson E., 2010; Atkinson L., Shaw R.L. et al., 2011; Joss, J., Howell M., 1989; Sigal Zilcha-Mano, Langer E. 2016; Maher J.H., Lowe J.B., 2015; Abrams L.S., Curran L., 2011; Martell L., 2001; Rassi A., Wattimena J., Black K., 2013; Hofmann S.G., Sawyer A.T., Witt A.A., 2010; Mommersteeg P.M., Van Beugen S. et al., 2013).

It is also important to note the presence of disparate studies in relation to prevention, awareness and their effectiveness in manifesting health-saving behavior in women of childbearing age and during pregnancy (Akerjordet K., Severinsson E., 2010; Lennon S.L., 2016; Greyson D.L., Johnson J.L., 2016; 
Abrams L., Curran L., 2011; Carolan M. 2007; Roth B., Robbins D., 2004; Dhillon A. et. Al., 2017; Suena H.M. et al., 2017; Maher J.H., Lowe J.B., 2015; Sakharova G.M., Antonov N.S.).

However, the study of reproductive health and maternity sphere is quite young, the theoretical model is still in its infancy and requires further confirmation of the experimental material, the development of preventive measures seems extremely relevant.

\section{Design}

The proposed research design complies with international standards for conducting preventive measures and evaluating their effectiveness. The materials developed for the implementation of the program of individual information for pregnant women can later be widely used in the professional activities of doctors, psychologists and social workers.

The obtained results can be applied to more effective psychological support for pregnant women, as well as within the framework of psychological counseling on a wide range of issues of reproductive health.

The aim of the research is to study the psychological readiness for motherhood (conscious motherhood) in connection with the health-saving behavior of pregnant women.

In connection with the goal, the following research objectives are formulated:

1) analysis of theoretical concepts and relationships between them (psychological readiness for motherhood, the subjective meaning of pregnancy, conscious motherhood, etc.);

2) the study of socio-demographic characteristics of pregnant women;

3) the study of the personal characteristics of pregnant women;

4) analysis of knowledge, attitudes and behavior of pregnant women in relation to various aspects of health-saving behavior (alcohol use, fetal alcohol syndrome, HIV and sexually transmitted infections, tobacco smoking, etc. in the framework of the "health beliefs" model);

5) the study of attitudes towards pregnancy and readiness for motherhood;

6) a comparative study of aspects of the health-saving behavior of a woman, her psychological readiness for motherhood and personal characteristics;

7) preventive measures (active and passive information) in the field of women's health, especially during pregnancy and immediately after childbirth;

8) evaluation of the effectiveness of preventive measures;

9) psychological support for pregnancy of women participating in the study.

\section{Expected results}

General expected results of the project:

1. Pregnant women of childbearing age do not have complete correct knowledge and adequate attitudes regarding aspects of health-saving behavior, thereby demonstrating problematic behavior (alcohol, tobacco smoking, the risk of alcohol- exposed pregnancy, that is, the risk of fetal alcohol syndrome).

2. The features of the components of the "health beliefs model" (knowledge-attitudes-behavior) are interrelated with the type of attitude towards pregnancy, psychological readiness for motherhood, as well as with the personality characteristics of pregnant women. The results extend the scope of this preventive model.

3. Under the influence of implemented preventive measures (active and passive information), the behavior of pregnant women becomes healthier, knowledge and attitudes in this sphere change.

4. The preventive materials developed by the project and the implemented measures prove their effectiveness and can be recommended for use in the area of health related to the reproductive sphere of women.

\section{Conclusions}

Applied importance can be determined by the possibility of using the results obtained in the framework of prevention and correction of behavior of pregnant women in order to give birth to a healthy baby, and in general, to maintain a high level of women's health, including the conditions of women's consultations and other medical and psychological institutions of the country, in particular in the sphere of reproductive health. Moreover, the results of the study can contribute to improving the performance of specialists in obstetric-gynecological and clinical-psychological profiles. 


\section{References}

Dobryakov I. V. Perinatal psychology. - SPb.: Piter, 2010. 234 p. (In Russian)

Matveeva E. V. Ways of educating readiness for motherhood and the quality of the mother's position in the Russian and American cultures of modern society. - Moscow - New York: 2004, pp. 11-30 (In Russian)

Meshcheryakova S. Yu. Psychological readiness for motherhood. Questions of psychology 5, 2000, pp. 18-27 (In Russian)

Nohr E.A., Vaeth M., Bech B.H., Henriksen T.B., Cnattingius S., Olsen J. Maternal obesity and neonatal mortality according to subtypes of preterm birth. Obstet Gynecol. 110 (5); 2007, p. 1083-1090.

Ovcharova R. Psychology of parenting: Proc. manual for students of higher educational institutions. Moscow: publishing center "Academy", 2005. 368 p. (In Russian)

Savenysheva S. S. Attitude to motherhood modern women // Herald of SPbSU. Series 12. Sociology. 2008. No. 4, pp. 45-55 (In Russian) 